\title{
Análise do diâmetro transverso do processo odontoide com uso da tomografia computadorizada
}

\author{
Analysis of the transverse diameter in the odontoid process by \\ computerized tomography \\ Análisis del diámetro transverso del proceso odontoides con uso de \\ la tomografía computarizada
}

\author{
Fábio Kazuo Soejima' \\ Carlos Henrique Maçaneiro² \\ Rodrigo Fetter Lauffer ${ }^{3}$ \\ Marco Antônio Rodacki ${ }^{4}$
}

\section{RESUMO}

Introdução: o diâmetro reduzido do odontoide é um desafio para o cirurgião no planejamento pré-operatório e escolha na fixação da fratura com um ou dois parafusos. Objetivo: analisar a medida do diâmetro transverso (DT) do processo odontoide por meio da tomografia computadorizada para o planejamento pré-operatório da osteossíntese com um ou dois parafusos. Métodos: foram analisadas 79 tomografias computadorizadas cervicais de pacientes adultos, sem deformidades congênitas ou queixas de dor em coluna cervical alta, atendidos em uma clínica privada de Blumenau - Santa Catarina. Resultados: as médias de idade não revelaram diferença estatisticamente significante entre os sexos, mas observou-se diferença estatisticamente significante entre as médias do DT do odontoide de homens e mulheres. A média do diâmetro transverso nos homens foi de 10,29 mm, e nas mulheres de 9,39 $\mathrm{mm}$. Conclusão: a tomografia computadorizada mostrou-se útil para a

\section{ABSTRACT}

Introduction: the reduced diameter of the odontoid is a challenge for the surgeon in preoperative planning and in choosing between the fixation of the fracture with one or two screws. Objective: to analyze the transverse diameter (TD) of the odontoid process by computerized tomography (CT) for preoperative planning of osteosynthesis with one or two screws. Methods: Seventynine cervical CT scans of adults without congenital malformations or complaints of pain in the upper cervical spine, treated at a private clinic in Blumenau - Santa Catarina, Brazil were analyzed. Results: the mean age revealed no statistically significant difference between the sexes, but there was a statistically significant difference between the average TD of the odontoid of men and women. The TD average in men was of $10.29 \mathrm{~mm}$, and in women it was of $9.39 \mathrm{~mm}$. Conclusion: computerized tomography proved to be useful for measuring the odontoid
\end{abstract}

\section{RESUMEN}

Introducción: el diámetro reducido de la odontoides es un desafio para el cirujano en la planificación pre-operatoria y de la elección en la fijación de la fractura con uno o dos tornillos. Objetivo: analizar la medida del diámetro transverso (DT) del proceso odontoides, por medio de la tomografía computarizada para el planeamiento pre-operatorio de la osteosíntesis con uno o dos tornillos. Métodos: fueron analizadas 79 tomografias computarizadas cervicales de pacientes adultos, sin deformidades congénitas o reclamos de dolor en la columna cervical alta, atendidos en una clínica privada de Blumenau - Santa Catarina, Brasil. Resultados: los promedios de edad no revelaron diferencia estadisticamente significante entre los sexos, pero se observó diferencia estadisticamente significante entre los promedios del DT de la odontoides de hombres y mujeres. El promedio del $D T$ en los hombres fue de 10,29 $\mathrm{mm}$, $y$ en las mujeres de 9,39 $\mathrm{mm}$. Conclusión: la tomografia computarizada se

\footnotetext{
Trabalho realizado em Blumenau (SC) em conjunto com o Instituto de Ortopedia e Traumatologia de Joinville - IOT - Joinville (SC), Brasil.

'Médico Ortopedista de Blumenau (SC), Brasil.

${ }^{2}$ Chefe do Serviço de Residência Médica do Instituto de Ortopedia e Traumatologia - IOT - Joinville (SC), Brasil.

${ }^{3}$ Preceptor do Serviço de Residência Médica do Instituto de Ortopedia e Traumatologia - IOT - Joinville (SC), Brasil.

${ }^{4}$ Médico Radiologista de Blumenau (SC), Brasil. 
medição do diâmetro transverso do processo odontoide. A fixação com um parafuso cortical de $3,5 \mathrm{~mm}$ seria possível em todos os casos analisados e a fixação com dois parafusos seria possível em $83,9 \%$ dos homens e $62,5 \%$ das mulheres. process. The fixation with a $3.5 \mathrm{~mm}$ cortical screw would be possible in all examined cases, and the fixation with two screws would be possible in $83.9 \%$ of the men and $62.5 \%$ of the women.

KEYWORDS: Spinal cord/surgery; mostró útil para la medición del diámetro transverso del proceso odontoides. La fijación con un tornillo cortical de 3,5 mm sería posible en todos los casos analizados y la fijación con dos tornillos sería posible en el 83,9\% de los hombres y el 62,5\% de las mujeres.
DESCRITORES: Coluna vertebral/ cirurgia; Processo odontoide/ lesões; Fixação de fraturas/ métodos; Parafusos ósseos; Tomografia computadorizada por raios $\mathrm{X}$ x-ray, computed

\author{
DESCRIPTORES: Columna \\ vertical/cirugía; Proceso \\ odontoides/lesiones; Fijación \\ de las fraturas/métodos; \\ Tornillos Óseos; Tomografía \\ computarizada por rayos $\mathrm{X}$
}

\section{INTRODUÇÃO}

As fraturas do processo odontoide correspondem de 5 a $15 \%$ das fraturas da coluna cervical ${ }^{1}$. As características dessa lesão, tais como incidência, epidemiologia, mecanismo de trauma, características clínicas e conduta terapêutica são discutíveis e podem variar de acordo com a idade do paciente ${ }^{2}$. O tratamento cirúrgico, por meio da osteossíntese com parafusos das fraturas tipo II de Anderson D'Alonzo ${ }^{3}$ em adultos, vem apresentando boa aceitação. No entanto, o diâmetro reduzido do odontoide apresentase como um desafio para o cirurgião no planejamento e escolha da fixação com um ou dois parafusos.

O objetivo deste estudo foi analisar a medida do diâmetro transverso (DT) do processo odontoide, por meio da tomografia computadorizada, para o planejamento préoperatório da osteossíntese com um ou dois parafusos.

\section{MÉTODOS}

Foram analisadas 79 tomografias computadorizadas cervicais de pacientes atendidos em uma clínica privada da cidade de Blumenau, Santa Catarina, a partir de Junho de 2008. Os pacientes foram informados da pequisa e assinaram o Termo de Consentimento Livre Informado. Todos os exames foram realizados com o aparelho TC Multislice, de 64 canais, da General Eletric ${ }^{\circledR}$ (GE), modelo VCT-XT. As medidas foram realizadas por um médico radiologista.

Os critérios de inclusão no estudo foram: pacientes adultos, sem deformidades congênitas ou queixas de dor em coluna cervical alta. As imagens tomográficas escolhidas para o estudo foram os cortes axiais perpendiculares ao processo odontoide e paralelos à articulação $\mathrm{C} 1-\mathrm{C} 2$, na área correspondente das fraturas tipo II de Anderson e D'Alonzo ${ }^{3}$, conforme proposto em um estudo relizado por Nucci et al. ${ }^{4}$.

$\mathrm{O}$ diâmetro tranverso (DT) entre as corticais externas do processo odontoide foi mensurado em milímetros (Figura 1). Foram calculados a média e o desvio padrão das medidas obtidas de uma forma total e, depois, estratificados por sexo para comparação.

\section{RESULTADOS}

Verificadas as suposições de normalidade (por meio dos testes de Kolmogorov-Smirnov - K-S - e Shapiro-Wilks - W $\mathrm{p}=0,844$ ) e igualdade de variância (Teste de Levene para igualdade de variâncias - Levene's Test of Equality of Variances - $\mathrm{p}$ $=0,732$ ), aplicou-se o teste $t$ para comparação das médias de idade e do DT. A média das idades $(\mathrm{p}=0,749)$ não revelou diferença estatisticamente significante entre os sexos, mas observou-se diferença estatisticamente significante entre as médias do DT do odontoide de homens e mulheres. $(p<0,001)$. A média do DT nos homens foi de 10,29 mm, e nas mulheres foi de 9,39 mm, conforme mostra o Box Plot da Figura 2.

As demais estatísticas calculadas para a amostra estão apresentadas na Tabela 1.

\section{DISCUSSÃO}

$\mathrm{O}$ adequado tratamento das fraturas do odontoide tem sido motivo de muitos estudos, e sua escolha depende da experiência de cada médico ${ }^{5-8}$. Dentre os três tipos de fraturas propostos pela classificação de Anderson e D'Alonzo ${ }^{3}$, o tipo II é o que apresenta mais controvérsias no tratamento.

O tratamento conservador com uso de halo-gesso, ou gesso minerva, classicamente descrito na literatura, apresenta taxas de consolidação muito distintas que variam de 5 a $63 \%$ na literatura ${ }^{9,10}$, e complicações tais como: soltura, infecção e deconforto grave dos pinos, úlceras de pressão, lesão nervosa, perfuração da dura-máter e disfagia ${ }^{11}$.

Fraturas do processo odontoide com desvio inicial $>5 \mathrm{~mm}$, angulação $>10^{\circ}$, desvio em dois planos, diástase dos fragmentos e idade acima de 40 anos são fatores atribuídos à falha no tratamento conservador ${ }^{12,13}$. Assim, a indicação para procedimento cirúrgico deve ser considerada.

A artrodese via posterior $\mathrm{C} 1-\mathrm{C} 2$, por meio das técnicas descritas por Gallie ${ }^{14}$ e Brooks ${ }^{15}$, tem sido utilizada há muito tempo e com bons resultados. No entanto, restringe os movimentos de rotação da coluna cervical em até $50 \%{ }^{1}$.

A osteossíntese das fraturas do odontoide com uso de dois parafusos foi descrita inicialmente por Böhler ${ }^{16}$ e mostrou-se 
vantajosa com relação à artrodese via posterior $\mathrm{C} 1-\mathrm{C} 2$, pois preserva o movimento de rotação. Segundo Schaffler et al. ${ }^{17}$, citado por Defino ${ }^{18}$, o diâmetro reduzido do odontoide $(7,4$ a $12,8 \mathrm{~mm}$ ) dificulta e, algumas vezes, não permite a colocação de dois parafusos. Nakanishi ${ }^{18}$, Pointllard et al. ${ }^{19}$ publicaram trabalhos com a utilização de um único parafuso e acreditam ser o suficiente para estabilizar a fratura. Nucci et al. ${ }^{4}$ realizaram uma avalição tomográfica do processo odontoide em adultos normais e observaram que não houve diferença estatisticamente significante entre os valores nos sexos masculino e feminino. Sugeriram que o diâmetro transverso mínimo de $9 \mathrm{~mm}$ seria fundamental para a utilização de dois parafusos corticais de 3,5 mm. Em 95\% dos casos estudados foi possível a colocação de dois parafusos.

Após análise dos dados coletados no presente trabalho e dos apresentados na literatura, observou-se que a relação entre o diâmetro tranverso do processo odontoide e a escolha do diâmetro do parafuso que será utilizado na osteossíntese são fundamentais para estabelecer opção de fixação com um ou dois parfusos. Nesse caso, discorda-se de Nucci et al. ${ }^{4}$, quando estes

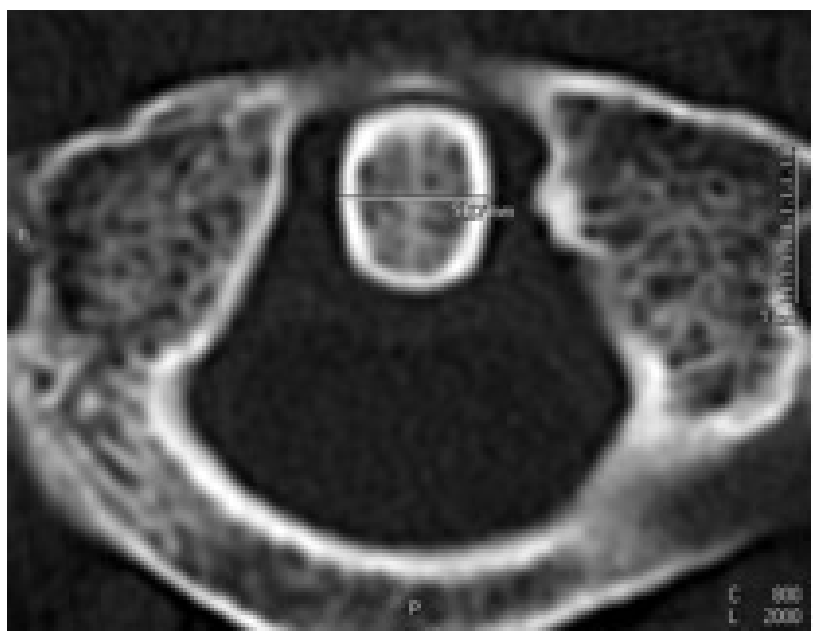

Figura 1

Corte axial de tomografia computadorizada realizando aferição do DT apontam que não há diferença significativa dos valores entre os sexos, já que se verificou, no presente trabalho, valores proporcionalmente maiores do DT no sexo masculino.

O trabalho corrobora com os estudos de Schaffler et al. ${ }^{17}$, Nakanishi ${ }^{18} \mathrm{e}$ Pointillart et al. ${ }^{19}$, uma vez que os valores reduzidos do DT do processo odontoide podem dificultar a utilização de dois parafusos. Os valores do DT do presente trabalho, comparados com os parâmetros propostos por Nucci et al. ${ }^{4}$ (diâmetro tranverso mínimo de $9 \mathrm{~mm}$ e parafusos corticais de $3,5 \mathrm{~mm}$ ), mostraram que $83,9 \%$ dos pacientes do sexo masculino e $62,5 \%$ do sexo feminino apresentavam DT suficiente para utilização de dois parafusos corticais de $3,5 \mathrm{~mm}$.

\section{CONCLUSÃO}

A tomografia computadorizada mostrou-se útil para a medição do diâmetro transverso do processo odontoide. A fixação com um parafuso cortical de 3,5 mm seria possível em todos os casos analisados. A fixação com dois parafusos corticais de 3,5 mm seria possível na maioria dos homens e das mulheres analisados neste estudo.

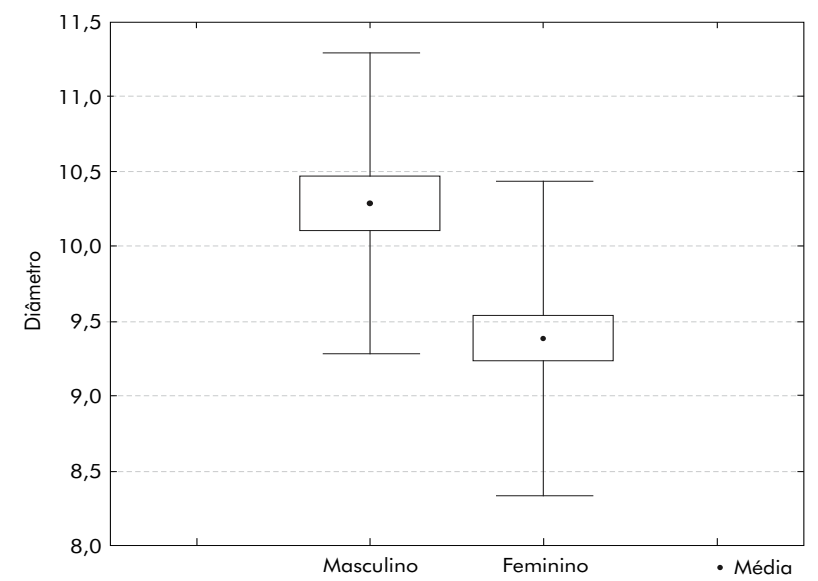

Figura 2

Box Plot com as medidas do diâmetro $(\mathrm{mm})$ da segunda vértebra da coluna cervial em 77 pacientes analisadas por tomografia computadorizada

TABELA 1 - Distribuição dos pacientes considerando Idade (em anos) e o Diâmetro Transverso (DT) do processo odontóide em milimetros, segundo sexo. Blumenau (SC), 2010.

\begin{tabular}{|c|c|c|c|c|c|}
\hline Variáveis/ Sexo & Média & Mediana & Desvio padrão & Valor mínimo & Valor máximo \\
\hline \multicolumn{6}{|l|}{ Masculino $(n=29 *)$} \\
\hline Idade (em anos) & $44,55^{\circ}$ & 43 & 12,95 & 26 & 73 \\
\hline Diâmetro transverso (DT) (em mm) & $10,29 b$ & 10,43 & 1,00 & 8,17 & 11,91 \\
\hline \multicolumn{6}{|l|}{ Feminino $(n=48)$} \\
\hline Idade (em anos) & $43,63^{a}$ & 41,5 & 11,85 & 25 & 74 \\
\hline Diâmetro transverso (DT) (em mm) & 9,396 & 9,36 & 1,05 & 7,11 & 11,8 \\
\hline \multicolumn{6}{|c|}{ Geral - masculino e feminino $(n=77)$} \\
\hline Idade (em anos) & 43,97 & 42 & 12,2 & 25 & 74 \\
\hline Diâmetro transverso (DT) (em mm) & 9,73 & 9,67 & 1,11 & 7,11 & 11,91 \\
\hline
\end{tabular}

$(\mathrm{n}=29)^{*}$ excluídos 2 com valores extremos dos demais

a diferença não significante $(p=0,749)$;

${ }^{b}$ diferença significante $(p<0,001)$. 


\section{REFERÊNCIAS}

1. Vaccaro AR, Betz RR, Zeidman SM. Cirurgia da coluna: princípios e prática. Rio de Janeiro: Dilivros; 2007. p. 441-62.

2. Defino HLA. Lesões traumáticas da coluna cervical alta. Rev Bras Ortop. 2002;37(4):99-107.

3. Anderson LD, D'Alonzo RT. Fractures of the odontoid process of the axis. $\mathrm{J}$ Bone Joint Surg Am. 1974;56(8):166374.

4. Nucci RC, Seigal S, Merola AA, Gorup J, Mroczek KJ, Dryer J, et al. Computed tomographic evaluation of the normal adult odontoid. Implications for internal fixation. Spine (Phila Pa 1976). 1995;20(3):264-70.

5. Clark CR, White AA 3rd. Fractures of the dens. A multicenter study. J Bone Joint Surg Am. 1985;67(9):1340-8.

6. Sherk HH. Fractures of the atlas and odontoid process. Orthop Clin North Am. 1978;9(4):973-84.

7. Müller EJ, Wick M, Russe O, Muhr G. Management of odontoid fractures in the elderly. Eur Spine J. 1999;8(5):360-5.
8. Defino HLA, Fuentes AER. Osteossíntese das fraturas do processo odontoide. Rev Bras Ortop. 1995;30(8):604-8.

9. Amyes EW, Anderson FM. Fracture of the odontoid process: report of sixty-three cases. AMA Arch Surg. 1956;72(3):377-93.

10.Blockey NJ, Purser DW. Fractures of the odontoid process of the axis. J Bone Joint Surg Br. 1956;38(4):794817.

11.Garfin SR, Botte MJ, Waters RL, Nickel VL. Complications in the use of the halo fixation device. J Bone Joint Surg Am. 1986;68(3):320-5.

12. Castro MG. Fraturas do odontoide. Clin Ortop. 2000;1(4):801-6.

13.Schatzker J, Rorabeck CH, Waddell JP. Fracture of the dens (odontoid process). An analysis of thirtyseven cases. J Bone Joint Surg Br. 1971;53(3):392-405.

14.Gallie WE. Fractures and dislocations of the cervical spine. Am J Surg. 1939;46:495-99.

15.Brooks AL, Jenkins EB. Atlanto-axial arthrodesis by the wedge compression method. J Bone Joint Surg Am. 1978;60(3):279-84.
16.Böhler J. Anterior stabilization for acute fractures and non-unions of the dens. J Bone Joint Surg Am. 1982;64(1):18-27.

17.Schaffler MB, Alson MD, Heller JG, Garfin SR. Morphology of the dens. A quantitative study. Spine (Phila Pa 1976). 1992;17(7):738-43.

18. Nakanishi T. Internal fixation of odontoid fracture. Orthop Trauma Surg. 1980;23:399-406.

19.Pointillart V, Orta AL, Freitas J, Vital JM, Senegas J. Odontoid fractures. Review of 150 cases and practical application for treatment. Eur Spine J. 1994;3(5):282-5.

\section{Correspondência}

Fabio Kazuo Soejima

Rua Armando Odebrecht, 70/910

CEP: 89020-403 - Blumenau (SC), Brasil

E-mail: fabiosoejima@hotmail.com 